\title{
A cohort study of elderly people in Bloemfontein, South Africa, to determine health-related quality of life and functional abilities
}

\author{
A M Gerber, ${ }^{1} \mathrm{PhD}$; R Botes, ${ }^{2} \mathrm{MSc} ;$ A Mostert, ${ }^{1} \mathrm{MB}$ ChB; A Vorster, ${ }^{1} \mathrm{MB} \mathrm{ChB}$; E Buskens, ${ }^{2} \mathrm{PhD}$ \\ ${ }^{1}$ Department of Basic Medical Sciences, Faculty of Health Sciences, University of the Free State, Bloemfontein, South Africa \\ ${ }^{2}$ Department of Health Technology Assessment, University Medical Centre Groningen, University of Groningen, Netherlands
}

Corresponding author: A M Gerber (gerberam@ufs.ac.za)

\begin{abstract}
Background. An ageing population has become an issue of global importance. According to statistics, the number of people aged $\geq 60$ years will outnumber children $<5$ years by 2020 .

Objective. To identify chronic and comorbid diseases that contribute to reduced quality of life (QoL) and functional ability in elderly people living in nursing homes in Bloemfontein, Free State, South Africa (SA).

Methods. This study used utility- and capability-based questionnaires EQ-6D and a modified ICECAP-O to identify chronic and comorbid diseases that contribute to reduced QoL and functioning in the elderly. An information leaflet was supplied to respondents, along with an informed consent form that each signed and dated. The respondents participated voluntarily and anonymously. Structured interviews were conducted. No algorithm for the EQ-6D or ICECAP-O is available for the SA population. Statistical Package for the Social Sciences version 16 was used to perform the sum score calculations. Data were presented using standard descriptive statistics (frequencies, medians, means, standard deviations and standard errors). Results. The total sample comprised 104 elderly respondents, $72.1 \%$ females and $27.9 \%$ males (mean age 77 years). Most suffered from at least two of the following diseases: hypertension $(68.8 \%)$, joint disease $(46.2 \%)$, heart disease $(22.1 \%)$, cancer (19.2\%) and psychological disorders (18.3\%). The EQ-6D indicated that 'pain' (48.3\%) and 'mobility' (36.2\%) were the domains chiefly affected. Elderly subjects with extreme problems reported all domains to be equally affected, with the exception of 'cognition' (29.1\%).

Conclusions. Our results confirm that diseases result in pain and affect mobility and cognition in old age. Access to healthcare and services for older people involves recognition of the importance of health promotion and activities that will help prevent disease, and there should be a focus on maintaining independence, prevention and delay of disease, and disability treatment. This includes improving QoL in elderly people with existing disabilities. Reform of medical care services is essential to improve healthcare for the elderly and thus improve their QoL.
\end{abstract}

S Afr Med J 2016;106(3):298-301. DOI:10.7196/SAMJ.2016.v106i3.10171

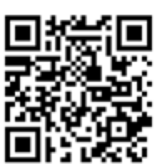

In April 2002, the United Nations Political Declaration and Madrid International Plan of Action on Ageing ${ }^{[1]}$ adopted 'a turning point in how the world addresses the key challenges of building a society for all ages'. The World Health Organization ${ }^{[2]}$ warns that effective strategies should be found to address the problems faced by an ageing world population, as chronic diseases will greatly affect the quality of life (QoL) of older people. People all over the world are living longer, levels of chronic illness are increasing, and a decrease in general wellbeing is poised to become a major global public health challenge. ${ }^{[2]}$

'An ageing world population refers to the process by which the older population becomes a proportionally larger component of the total population. This is an outcome of a population's demographic transition from higher to lower levels of fertility and mortality. ${ }^{[3]}$ The increase in longevity has largely been due to the decline in deaths from cardiovascular disease, i.e. stroke and ischaemic heart disease, mainly because of improved health management and effective health interventions.

Effective health interventions at the right time can increase life expectancy. Worldwide, life expectancy of older people continues to rise. According to statistics, by 2020 the number of people aged $\geq 60$ years will outnumber children $<5$ years of age, and by 2050 , the world's population aged $\geq 60$ years is expected to total 2 billion, from 841 million today. Eighty percent of these older people will be living in low-income and middle-income countries. 'It is therefore ironic that a relative lack of concern is observed in many of the less developed countries in the world. ${ }^{[3]}$
Although people are living longer, they are not necessarily healthier. Nearly a quarter (23\%) of the overall global burden of death and illness is in people aged $>60$ years. This burden is attributable to diseases such as cancer, chronic respiratory diseases, heart disease, musculoskeletal diseases such as arthritis and osteoporosis, and mental and neurological disorders.

Sub-Saharan Africa carries a high disease burden, and the elderly population in this region is set to increase from 36.6 million to 141 million by $2050{ }^{[4]}$ Multimorbidity causes a cumulative decline in self-rated health in the elderly. Social networks are complex interactions between family, the wider community and spouses to determine mental and physical health. ${ }^{[5]}$ Different subgroups in the elderly population experience health and health-related QoL very differently. ${ }^{[6]}$

Healthcare and treatment choices for the elderly are challenges made unique by various social, psychological and physical problems occurring in advanced age. ${ }^{[7]}$ Factors such as resilience, self-efficacy and the perception of life as meaningful and manageable are important factors to consider when determining QoL and function in this age group ${ }^{[8]}$ Older people should continue to participate in the economic, social, cultural and political lives of their societies and enjoy health, security and fulfilment. ${ }^{[1]}$

In terms of the capability approach, functional ability is important, focusing on what people actually achieve with the resources at their disposal. ${ }^{[9]}$ Different diseases affect an individual's ability to function to different degrees, and can induce a state referred to as potential 
disability. In order to make appropriate healthcare and treatment choices, it is therefore essential to understand how health profiles affect QoL and function in the elderly. However, premorbid QoL, age and comorbidity all also have significant effects. ${ }^{[10]}$ Statistics in Europe indicate that two-thirds of European elderly people report disability. ${ }^{[6]}$ According to Unger, ${ }^{[1]]}$ it is more than likely that the approach to healthcare for the elderly will change significantly in the future, with more focus on self-care initiatives, e-health systems and patient-centred approaches, whereas Arai et al. ${ }^{[7]}$ suggest that reform of medical care services is essential for better healthcare delivery to the elderly to improve their QoL.

To enable us to adapt healthcare services, it is important to prioritise the personal needs of the elderly regarding resource allocation and healthcare services. ${ }^{[12]}$ Medical decision-making should always be a shared interaction between patient and medical staff. Elderly patients with multiple chronic conditions report a desire for individualised patient-centred care. The elderly patient will have to make medical treatment choices based on personal values, beliefs, goals, treatment options, treatment benefits/risks and costs. ${ }^{[13]}$

This study investigated the effects of prevalent chronic diseases and multimorbidity on functional ability and QoL in elderly people in Bloemfontein, South Africa (SA). We expected that elderly individuals suffering from multimorbidity would experience a larger decline in QoL and functional achievement than those suffering from a single chronic disease. We hope that this information can provide an insight into the experiences of the diseased elderly, and thus contribute to a much-needed change in the approach to healthcare and management of this age group.

\section{Objective}

Using utility- and capability-based questionnaires, to identify chronic and comorbid diseases that significantly contribute to the reduction of QoL and function in the elderly.

\section{Methods}

We recruited 104 elderly individuals living in Bloemfontein. The respondents were all aged $\geq 65$ years, were living in nursing homes, and were dependent and receiving moderate care. Individuals who were unwilling or unable to complete the questionnaires were excluded.

\section{Data collection}

The following procedure was followed: the investigators identified themselves and described the purpose and relevance of the study to potential subjects, who were then given an information leaflet and an informed consent form to sign and date. The subjects were asked if they were willing to participate voluntarily and anonymously in the completion of the questionnaires and the evaluations, which would take about 15 minutes. Finally, the investigators assured respondents that their identity and privacy would be maintained if the findings of this study were published.

Respondents were asked to describe their own health using the descriptive system of the Six-Dimensional EuroQol questionnaire (EQ-6D) and a version of the ICEpop capability measure for older people (ICECAP-O).

\section{The EQ-6D}

The EQ-6D is a utility-based questionnaire developed by the Euroqol group. It mainly focuses on health-related QoL. Domains included in the EQ-6D are mobility, self-care, usual activities, pain/discomfort, anxiety/depression and cognition. The EQ-6D is an updated version of the EQ-5D, including a sixth domain, namely cognition. Each domain has three possible answer categories: $1=$ 'no problem', $2=$ 'moderate problems' and 3 = 'extreme problems'. This part of the EQ-6D was used to describe the actual self-reported health state of the elderly respondents.

\section{ICECAP-O}

The ICECAP-O is a capability-based questionnaire, designed specifically for the elderly. The ICECAP-O version we used is different from the original questionnaire in that it contains five possible answer categories instead of four. These are attachment (feelings of love and affection), enjoyment (activities providing joy or pleasure), security (feeling secure when considering health and finances), role (having a purpose) and control (making one's own decisions) ${ }^{[14,15]}$ In the context of this study the five outcomes were viewed as being functions and not capabilities. The five answer categories are as follows: $1=$ all functions are achieved, $2=$ a lot are achieved, $3=$ some are achieved, $4=$ few are achieved, and $5=$ none are achieved. The respondents were instructed to complete the questionnaire by reflecting on their own health.

\section{Evaluation}

In addition, respondents were asked to evaluate 10 hypothetical health states for each questionnaire. This involved imagining the value they would apply to hypothetical states in the different domains. A visual analogue scale (VAS) was used for this valuation exercise, zero (0) representing the worst imaginable health state and 100 the best imaginable health state. Since there are six domains in the EQ-6D questionnaire, each health state consisted of six consecutive numbers, as follows: 111111, 112112, 212111, 111221, 212121, 133113, 212321, 333211, 323331 and 333333.

An identical version of the VAS scale was used in the evaluation of the ICECAP-O questionnaire. The health states evaluated from the ICECAP-O were 11111, 11122, 11245, 11312, 12335, 21114, 33333, 33544,44433 and 55555.

Demographic information and information on disease prevalence was collected, and structured interviews were conducted.

\section{Ethical approval}

The University Medical Centre Groningen (UMCG) gave ethical approval for the study, as it was initially part of a bigger study that involved the Dutch population. During the ethical approval of the pilot study, conducted in SA and the Netherlands, the UMCG ethical committee found that the interviews were not classified as invasive interventions, and no additional ethical application was warranted. No additional ethical approval was sought in SA. Each respondent received an information leaflet as well as a document regarding informed consent. They all signed and dated these documents.

\section{Statistical analysis}

The proportion of respondents reporting problems was calculated according to each of the EQ-6D and ICECAP-O domains. The EQ-6D sum scores, as well as the sum scores of the two questionnaires, were also calculated. The overall scores were calculated using the Dolan (UK) EQ-5D algorithm.

No algorithm for the EQ-6D or ICECAP-O is available for the SA population. Statistical Package for the Social Sciences version 16 was used to perform the sum score calculations. The data were analysed and interpreted by the investigators with the assistance of a statistician. Data were presented using standard descriptive statistics, e.g. frequencies, medians, means, standard deviations and standard errors.

Trends, correlations and meaningful differences were noted and interpreted to draw consequential conclusions. 


\section{Results}

The total sample comprised 104 respondents, of whom 75 (72.1\%) were females. The mean age was 77 years (range 74 - 92) (Table 1). Responses indicated that $16.3 \%$ had a primary school education, $50.0 \%$ a secondary school education and $18.3 \%$ a tertiary education, and that $74.0 \%$ were religious. The disease profiles showed that most subjects suffered from two or more comorbid illnesses, the five most prevalent being hypertension, joint disease, heart disease, cancer and psychological disorders.

Table 2 shows the EQ-6D domains most affected in the elderly subjects. The respondents experiencing 'some problems' rated the domains from the most to the least affected as follows: pain, mobility, cognition, anxiety, activity and self-care. Elderly subjects with 'extreme problems' reported all the domains to be equally affected, with the exception of 'cognition'.

Average values for the EQ-6D health states as evaluated by the respondents are shown in Fig. 1. There is very little variation between the health states, with values of between 72 and 75 .

Table 3 shows that the modified ICECAP-O descriptions indicating 'some' satisfaction showed that the 'role', 'security/pleasure' and 'attachment' functions were most affected. The descriptions indicating 'a little' satisfaction showed that 'pleasure', 'role' and 'security' were the functions most affected, while the descriptions indicating 'none' showed 'control' and 'role' to be most affected.

The average ICECAP-O health state evaluations of the elderly respondents showed values of between 73 and 76 (Fig. 2). Very little variance is seen between the health states.

\section{Discussion}

Respondents reporting some problems in the EQ-6D questionnaire indicated 'pain' and 'mobility' to be the domains most affected. However, the 'extreme' group reported the same amount of problems across all the domains with the exception of 'cognition. The general response indicated that 'pain', 'mobility' and 'cognition' were the domains most affected. This is not surprising, since the two most prevalent diseases in this sample were hypertension and joint disorders. We therefore speculate that suffering from hypertension and joint disorders could possibly account for the majority of the group reporting 'some problems' regarding pain and mobility. We furthermore suggest that the elderly experiencing 'extreme problems' are experiencing such a decline in health that QoL in general is affected.

The respondents' evaluations of the different health states indicate that the better and worse health states are valued similarly. It is difficult to determine which health states are implicitly more acceptable to the elderly and which are more unacceptable. An explanation could be that only $18.3 \%$ of the elderly respondents suffered from no or just one disease, while the rest were all suffering from more than one. Multimorbidity and the negative effects on selfrated health could account for this phenomenon, but further research is needed to confirm this finding.

The ICECAP-O results indicate that the same three functions were affected in the 'some' and 'a little' groups, security, pleasure and role being identified as the functions most affected. The 'none' group also reported role and control as functions affected. Intriguingly, the role 'function' is relevant in all three groups. We therefore speculate that although the majority of the respondents achieved high levels of wellbeing, some experienced difficulty in identifying with their new environment and position in society. These results further support the fact that although the elderly are all part of the same age group, levels of functional achievement subjectively separate them into distinct groups, with lack of achievement causing specific and unique problems. Because the respondents' evaluations of the
Table 1. Demographic data of the elderly subjects $(N=104)$ according to the EQ-6D

\begin{tabular}{ll}
\hline & $\boldsymbol{n}(\%)$ \\
\hline Gender & \\
Male & $29(27.9)$ \\
Female & $75(72.1)$ \\
Level of education & \\
Primary education & $17(16.3)$ \\
Secondary education & $52(50.0)$ \\
Diploma & $19(18.3)$ \\
University degree & $16(15.4)$ \\
Other & - \\
Religious & \\
Yes & $77(74.0)$ \\
No & $27(26.0)$ \\
Number of diseases & \\
None & $5(4.8)$ \\
1 & $14(13.5)$ \\
2 & $44(42.3)$ \\
3 & $17(16.3)$ \\
4 & $11(10.6)$ \\
5 & $8(7.7)$ \\
6 & $5(4.8)$ \\
\hline$i s e$
\end{tabular}

Disease type ${ }^{*}$

Hypertension

Joint disorders

Heart disease

Cancer

Psychological disorder

COPD

Kidney/gallstones

Stroke

Diabetes

Kidney disorder

Epilepsy

COPD $=$ chronic obstructive pulmonary diseas ${ }^{*}$ Respondents could list more than one disease.

Table 2. EQ-6D results per domain $(\%)(N=104)$

\begin{tabular}{llll}
\hline Domain & No problems & Some problems & Extreme problems \\
\hline Mobility & 61.4 & 36.3 & 7.2 \\
Self-care & 90.1 & 7.3 & 7.2 \\
Activity & 80.4 & 17.1 & 7.1 \\
Pain & 48.4 & 48.2 & 8.4 \\
Anxiety & 69.2 & 28.1 & 7.1 \\
Cognition & 74.3 & 29.1 & 1.3
\end{tabular}

different health states indicated little variance between the better and worse states, we argue that multimorbidity plays an important role in determining health state evaluations. 


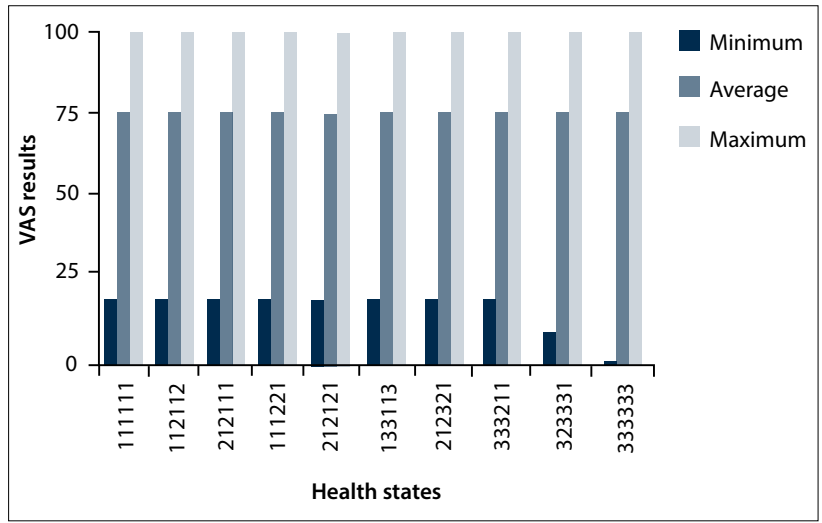

Fig. 1. The EQ-6D VAS evaluation results $(\mathrm{N}=104)$.

\begin{tabular}{llllll}
\multicolumn{7}{l}{ Table 3. ICECAP results $(\%)(\boldsymbol{N}=\mathbf{1 0 4})$} \\
\hline Domain & All & A lot & Some & A little & None \\
\hline Attachment & 82.2 & 11.1 & 9.3 & 2.3 & 0.3 \\
Pleasure & 42.4 & 38.1 & 15.4 & 9.2 & 0.2 \\
Security & 61.4 & 23.2 & 15.4 & 5.3 & 0.3 \\
Role & 46.1 & 31.2 & 18.4 & 7.1 & 2.4 \\
Control & 74.4 & 17.4 & 6.1 & 4.4 & 3.3
\end{tabular}

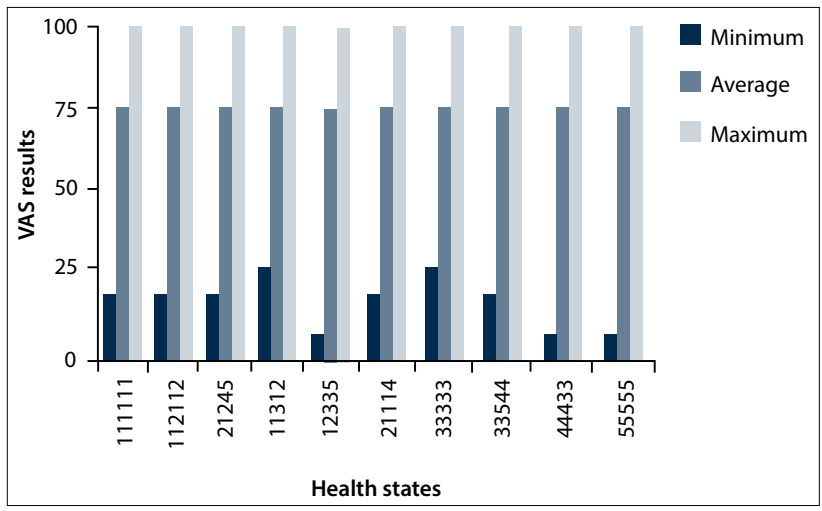

Fig. 2. The ICECAP VAS evaluation results $(\mathrm{N}=104)$.

The demographic ageing of a population is directly related to fundamental changes in its health and disease patterns. We aimed to provide policy makers with information that will help them make decisions regarding prioritisation and resource allocation for the elderly in the future. In addition, general practitioners, gerontologists and geriatric specialists can use this information to identify and assist elderly individuals at risk of declining health-related QoL and impaired functional achievement. Information also needs to be conveyed to the elderly individuals themselves to help them formulate personal, informed and effective healthcare choices. ${ }^{[3]}$

\section{Study limitations}

This study did not differentiate between specific disease types and their individual effects on health-related QoL. Scope exists for a more disease-orientated approach in future studies. The study population was limited to old-age homes in Bloemfontein.

\section{Conclusions}

An epidemiological transition under way all over the world indicates a shift from a predominance of infectious and parasitic diseases to one of chronic and degenerative diseases. ${ }^{[1]}$ Having more older people than ever before may imply increases in the prevalences of chronic disease and disability. From this study we conclude that policy makers and health services should look at designing interventions to address the onset and management of multimorbidity. Furthermore, integrated cost-effective interventions aimed at improving specific healthrelated domains as well as non-health-related functions according to health profiles should have a positive impact on QoL and functional achievement in the elderly. Our findings show that pain and mobility were the domains most affected. The functions of security, pleasure and especially role were not achieved to satisfaction, which has a profound effect on an individual's QoL and wellbeing. Addressing these problems through cost-effective health and psychological support will ensure optimal functioning and QoL achievements. Planning, policy development and allocation of resources to the ageing population are particularly difficult in developing countries, since populations often age before significant socioeconomic development has taken place. Advancing age will increasingly demand long-term, chronic, frail and end-of-life care, and at the same time increase the need for appropriately trained healthcare staff and facilities.

According to Dr John Beard, Director of Ageing and Life Course at the World Health Organization, 'Deep and fundamental reforms of health and social care systems will be required. ${ }^{[2]}$

Acknowledgement. We thank all who were willing to participate in the study and Ms T Mulder, medical editor in the Faculty of Health Sciences, University of the Free State, for technical and editorial assistance with preparation of the manuscript.

\section{References}

1. United Nations. Political declaration and Madrid International Plan of Action on Ageing. Second World Assembly. Madrid, Spain: United Nations, 2002. http://www.un.org/en/events/pastevents/pdfs/ Madrid_plan.pdf (accessed 2 February 2015)

2. World Health Organization. Ageing well' Must Be a Global Priority. News release. Geneva: WHO, 2. World Health Organization. 'Ageing well' Must Be a Global Priority. News release. Geneva: WHO,
6 November 2014. http://www.who.int/mediacentre/news/releases/2014/lancet-ageing-series/en/ (accessed 4 February 2015

3. Joubert J, Bradshaw D. Population ageing and health challenges in South Africa. In: Steyn K, Fourie J, Temple N, eds. Chronic Diseases of Lifestyle in South Africa: 1995-2005. Cape Town: South African Medical Research Council, 2006:204-219.

4. Kimokoti RW, Hamer DH. Nutrition, health, and aging in sub-Saharan Africa. Nutr Rev 2008;66(11):611-623. [http://dx.doi.org/10.1111/j.1753-4887.2008.00113.x]

5. Alonso-Morán E, Nuño-Solinís R, Orueta JF, Fernandez-Ruanova B, Alday-Jurado A, GutiérrezFraile E. Health-related quality of life and multimorbidity in community-dwelling telecare-assisted elders in the Basque Country. Eur J Intern Med 2015;26(3):169-175. [http://dx.doi.org/10.1016/j. ejim.2015.02.013]

6. König HH, Heider D, Lehnert T, et al. Health status of the advanced elderly in six European countries: Results from a representative survey using EQ-5D and SF-12. Health Qual Life Outcomes 2010;8:143. http://hqlo.biomedcentral.com/articles/10.1186/1477-7525-8-143 (accessed 4 February 2010;8:143. http://hqlo.biomedcentral.com/articles/10.1186/1477-7525-8-143 (accessed 4 February
2016).

7. Arai H, Ouchi Y, Yokode M, et al. Toward the realization of a better aged society: Messages from gerontology and geriatrics. Geriatr Gerontol Int 2012;12(1):16-22. [http://dx.doi.org/10.1111/j.14475594.2011.00776.x

8. Bowling A, Iliffe S. Psychological approach to successful ageing predicts future quality of life in older adults. Health Qual Life Outcomes 2011;9:13. [http://dx.doi.org/10.1186/1477-7525-9-13]

9. Anand P. Capabilities and health. J Med Ethics 2005;31(5):299-303. [http://dx.doi.org/10.1136/ jme.2004.008706]

10. Van Jaarsveld CH, Sanderman R, Ranchor AV, Ormel J, van Veldhuisen DJ, Kempen GI. Genderspecific changes in quality of life following cardiovascular disease: A prospective study. J Clin Epidemiol 2002;55(11):1105-1112. [http://dx.doi.org/10.1016/S0895-4356(02)00506-1]

11. Unger F. Health is wealth: Considerations to European healthcare. Prilozi 2012;33(1):9-14.

12. Diederich A, Swait J, Wirsik N. Citizen participation in patient prioritization policy decisions: An empirical and experimental study on patients' characteristics. PLoS One 2012;7(5):e36824. [http:// empirical and experimental study on patiens
dx.doi.org/10.1371/journal.pone.0036824]

13. Holzmueller CG, Wu AW, Pronovost PJ. A framework for encouraging patient engagement in medical decision making. J Patient Saf 2012;8(4):161-164. [http://dx.doi.org/10.1097/ in medical decision

14. Coast J, Flynn TN, Natarajan L, et al. Valuing the ICECAP capability index for older people. Soc Sci Med 2008;67(5):874-882. [http://dx.doi.org/10.1016/j.socscimed.2008.05.015]

15. Coast J, Peters TJ, Natarajan L, Sproston K, Flynn T. An assessment of the construct validity of the descriptive system for the ICECAP capability measure for older people. Qual Life Res 2008;17(7):967976. [http://dx.doi.org/10.1007/s11136-008-9372-z]

Accepted 6 October 2015. 